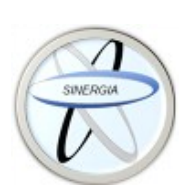

Revista Médica Sinergia

Vol. 6, Núm. 6, junio 2021, e684

\title{
Reconocimiento clínico de la enfermedad de Hansen
}

\section{Clinical recognition of Hansen's disease}

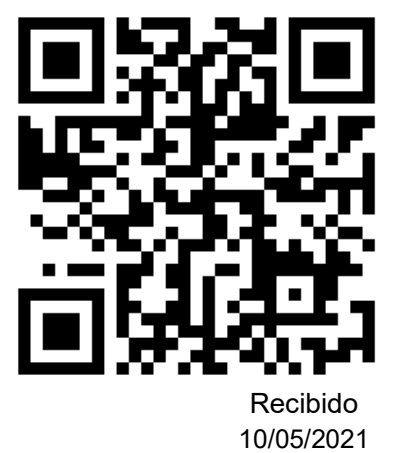

\author{
${ }^{1}$ Dr. Jorge Borbón Guevara \\ Hospital San Vicente de Paul, Heredia, Costa Rica \\ (iD https://orcid.org/0000-0003-3001-5935
}

${ }^{2}$ Dra. Ana Victoria Vargas Cruz

Área de Salud Valverde Vega, Alajuela, Costa Rica

(D) https://orcid.org/0000-0002-7703-4079

Corregido

$18 / 05 / 2021$
Aceptado

20/05/2021

\section{RESUMEN}

La enfermedad de Hansen o también conocida como Lepra, es una enfermedad crónica, infecciosa, transmisible, producida por el agente Mycobacterium leprae y Mycobacterium lepromatosis. Presenta un periodo de incubación en promedio de 5 años con un rango que va desde 1 a 20 años. Fue declarada un problema global mundial en el año 2000 y persiste con un numero constante de casos diagnosticados por año. Su vía de transmisión aún no se encuentra completamente dilucidada, las secreciones de las vías respiratorias superiores son la ruta de transmisión más común conocida hasta ahora. La presentación clínica y clasificación varían extensamente y estas dependen de la respuesta inmunológica del paciente a la infección. Actualmente se utilizan dos clasificaciones para agruparla: OMS y Ridley-Jopling. Es de gran importancia el reconcomiendo temprano de las lesiones y la educación continua sobre las mismas ya que permite un diagnóstico a tiempo, evitando el desarrollo de discapacidades.

PALABRAS CLAVE: Enfermedad de Hansen; lepra; Mycobacterium leprae.

\section{ABSTRACT}

Hansen's disease also known as Leprosy, is a chronic, infectious, transmissible disease caused by the agents Mycobacterium leprae and Mycobacterium lepromatosis. It has an incubation period of 5 years on average and age range that goes from 1 to 20 years. It was declared a global problem in 2000 and persists with a constant number of diagnosed cases per year. Its transmission route is not yet fully elucidated. Upper respiratory secretions are the most common transmission route known so far. The clinical presentation and classification vary and these depend on the patient's immune response to infection. Two classifications are currently used to group it: OMS and Ridley-Jopling. Early recognition of injuries and continuing education 
about them is of great importance since it allows a diagnosis in time, avoiding the development of disabilities.

KEYWORDS: Hansen's disease; leprosy; Mycobacterium leprae.

${ }^{1}$ Médico general, graduado de la Universidad de Costa Rica (UCR). Cód. MED16422. Correo: jiborbon@gmail.com

${ }^{2}$ Médica general, graduada de la Universidad de Ciencias Médicas (UCIMED). Cód. MED16150. Correo: vargasav07@gmail.com

\section{INTRODUCCIÓN}

La enfermedad de Hansen es una patología que afecta predominantemente a la piel y los nervios periféricos, ocasionada por la infección del agente Mycobacterium leprae (M. leprae) o Mycobacterium lepromatosis (M. lepromatosis) (1) reconocido como nueva especie involucrada a partir del 2018 (2), que resulta en neuropatía y consecuencias asociadas a largo plazo, incluidas deformidades y discapacidad (3) con una vía de transmisión que aún no se encuentra completamente definida, siendo este último el principal factor limitante en su erradicación. Mediante la presente revisión bibliográfica se busca capacitar al personal de salud $y$ otorgarle las herramientas necesarias para generar una sospecha clínica con el propósito de detectar la enfermedad en su estadio temprano y con esto difundir un impacto en la erradicación de la enfermedad.

\section{MÉTODO}

Para la realización de este artículo de revisión bibliográfica se consultaron un numero de dieciséis artículos, entre estos: artículos de revisión bibliografía, revisiones de caso, y artículos de investigación, con una antigüedad no mayor a cinco años, en cada uno de ellos, determinada por el año de publicación. Las bases de datos utilizadas con esta finalidad fueron Pubmed y Uptodate principalmente. Se consultaron las directrices internacionales de la Organización Mundial de la Salud (OMS), con los últimos objetivos establecidos para la erradicación de la enfermedad.

Se emplearon como términos de búsqueda las palabras clave: "hansen clínica", "lesiones leprosas", "exclusión por lepra", "secuelas lepra", "diagnostico Hansen", "lesiones por micobacterias". Todas las revisiones utilizadas son de relevancia medico científica, presentes en idioma inglés y español. Se seleccionaron aquellos artículos que describieran ampliamente las características clínicas de la enfermedad y que reflejaran la importancia de su reconocimiento temprano.

\section{EPIDEMIOLOGÍA}

La enfermedad de Hansen es una de las patologías de mayor antigüedad en la historia de las condiciones epidémicas en humanos (4), considerada una afección tropical, endémica de los continentes de América, Asia y África (1), se remontan al ciclo $\mathrm{V}$ a.C los hallazgos que describen las características de las lesiones cutáneas desfigurantes de la enfermedad no tratada (4). En el año 1873 (5), en Noruega, el Dr. Amauer Hansen descubre el M. Leprae (4), convirtiéndose en la primera bacteria identificada como agente causal de una enfermedad (6).

Es declarada endémica en más de 140 países alrededor del mundo, con una mayor incidencia desde el 2015 en países como India, Brazil e Indonesia (4), en el año 1991 la OMS estableció la ambiciosa meta de eliminar la enfermedad para el año 2000 (4), el éxito de las medidas de control 
instauradas ha sido revolucionario, gracias a la implementación de la terapia con múltiples fármacos $(4,7)$; pero a pesar de haber sido declarada eliminada como un problema global mundial (definido como una prevalencia puntual por debajo de 1 por 10 000 habitantes) en el año 2000, el número de pacientes nuevos diagnosticados con lepra sigue siendo significativo, con más de 200000 casos en el 2016 (3), manteniéndose este número constante a lo largo de los últimos años $(4,7)$.

En Costa Rica desde el año 2015 se diagnostica un promedio de 10 casos por año (8).

Es de importancia mencionar que un $6 \%$ de los casos al momento del diagnóstico, se encuentran en fases avanzadas de la enfermedad asociadas a déficits neurológicos visibles con mutilaciones (7). Para el año 2016 la OMS definió tres objetivos con el fin de eliminar la enfermedad, bajo el lema "2016-2020 acelerado hacia un mundo sin lepra", estos objetivos fueron la promoción de medidas gubernamentales que promovieran un diagnóstico temprano, prevención de discapacidades e inclusión de personas afectadas a la vida pública para combatir la discriminación $(4,7,9)$.

\section{TRANSMISIÓN}

Enfermedad crónica infecciosa causada por el complejo Mycobacterium lepromatosis el cual está conformado por los agentes $M$. leprae y de más reciente diagnostico $\mathrm{M}$. lepromatosis (1).

M. leprae es un organismo acido-resistente de crecimiento lento obligado, que no puede ser cultivado en medios artificiales (4), solamente crece en patas de ratones y en el armadillo de nueve bandas (4), muestra predilección por la replicación en macrófagos, células endoteliales y células de Schwann (7) y las temperaturas bajas facilitan su crecimiento, crece de forma lenta y se divide una vez cada 12 días (4).

Ambos bacilos leprosos causan condiciones patológicas similares (1), pero su vía de transmisión aún no está completamente entendida $(4,1)$.

El mecanismo de transmisión depende de la infectividad del huésped y de la proximidad, frecuencia y duración del contacto. Las secreciones de las vías respiratorias superiores son la ruta de transmisión más común conocida, rara vez se ha informado contaminación cutánea o transmisión vertical (7). Existe amplia evidencia que indica un mayor riesgo de contagio para aquellas personas que presentan contacto directo con pacientes infectados por la Micobacteria, por medio de la diseminación de la partícula en medio de aerosol, al toser o estornudar (1). Se requieren de más investigaciones para esclarecer la vía de transmisión y las reservas naturales de los patógenos de la enfermedad. En el continente americano el armadillo de nueve bandas se ha establecido como huésped natural y reservorio de $M$. leprae y recientemente en el 2016 se determinó la presencia del agente en Ardillas Rojas (1). Las rutas de transmisión de las reservas animales posiblemente estén interrelacionadas con el medio ambiente en un ciclo ecológico elaborado que aún permanece sin descifrar (1). Lo cual puede explicar la constante incidencia mundial de la lepra y lo que parece constituir el paradigma para la OMS, en la eliminación mundial de la lepra, ya que su erradicación se basa enteramente en la transmisión de persona a persona y no aborda la transmisión zoonótica (7).

La susceptibilidad de contraer la enfermedad es altamente variable y multifactorial, entre los factores de riesgo podemos encontrar:

- Contacto cercano prolongado con pacientes contagiados en especial con los subtipos: lepra lepromatosa (LL), multibacilar (MB), o con exposición a 
armadillos (mascotas, personal de zoológicos, veterinarios)

- Rango de edad entre los 5-15 años y mayores de 30 al momento de la exposición

- Inmunosupresión, inmunodeficiencia, predisposición genética (7)

- Hacinamiento (6)

- Transmisión de la madre al feto (6)

- Tatuajes (6)

Factores de riesgo que involucran una alta probabilidad de desarrollar discapacidades físicas, son:

- Sexo masculino $(7,10)$

- Lepra lepromatosa (7)

- Reacciones leprosas inmunológicas (7)

Posterior al contagio el periodo de incubación suele oscilar entre 3 a 5 años para la lepra tuberculoide y entre 9 y 12 años para la lepra lepromatosa (7), generando manifestaciones clínicas en un 5-10\% de los pacientes infectados (4).

\section{CLÍNICA}

La variabilidad clínica de la enfermedad está determinada por el tropismo del agente en la piel y en el tejido nervioso periférico, la susceptibilidad genética e individual al $M$. leprae (4).

La morfología de las lesiones en la piel está determinada por cada variante clínica (4). Existen importantes diferencias en número y patrón de distribución de las lesiones (4). Cuando se tienen resultados positivos de biopsia en piel o tejido nervioso periférico, la enfermedad puede ser clasificada, basada en la histopatología de la muestra examinada que refleja el grado de respuesta inmune del paciente (11).

Existen dos clasificaciones para determinar la presentación clínica: la clasificación de Ridley - Jopling y la clasificación de la OMS, ambas son usadas para agrupar las diferentes variantes clínicas y guiar el diagnóstico:

1. Ridley - Jopling: combina manifestaciones clínicas, hallazgos histopatológicos y índices bacteriológicos.

2. OMS: se basa en el índice bacteriológico o el número de lesiones (7).

El sistema de clasificación de la OMS, ha demostrado ser eficaz para la clasificación clínica, el diagnóstico y tratamiento de la enfermedad (4), se basa en el índice bacteriológico $\mathrm{o}$ el número de lesiones cutáneas, cuando no se dispone de un frotis de piel (SS), los pacientes se clasifican como lepra paucibacilar (PB) cuando el número de lesiones cutáneas es de 1-5 y como MB cuando el número de lesiones cutáneas es 5 , sin embargo cuando se dispone de un examen SS, los pacientes con 1-5 lesiones cutáneas son clasificados como PB cuando la baciloscopia (BI) es negativa en todos los sitios examinados o MB cuando el $\mathrm{BI}$ es positivo en cualquier sitio examinado (7).

La presentación clínica y clasificación varían extensamente y estas depende de la respuesta inmunológica del paciente a la infección (7).

La piel, el sistema nervioso y el sistema reticuloendotelial son los principales involucrados, pero sin embargo otros sistemas pueden verse afectados como lo son el SRS, huesos y articulaciones, ojos, testículos, gandulas adrenales.

La clasificación Ridley - Jopling aparte de las dos formas polares: tuberculoide (paucibacilar) y lepromatosa (multibacilar), incluye una etapa intermedia amplia y se establece el subtipo basado en la proximidad clínica e histológica a una de las dos formas polares, el intermedio está conformado por tres subgrupos y ayuda a categorizar los diferentes tipos de lepra a lo largo del espectro (7):

1. Lepra tuberculoide polar (TT)

2. Lepra limítrofe tuberculoide (BT) 
3. Lepra limítrofe media (BB)

4. Lepra limítrofe lepromatosa $(\mathrm{BL})$

5. Lepra Lepromatosa polar (LL)

6. Lepra indeterminada (IL)

El diagnóstico de IL se utiliza para clasificar a aquellos pacientes que aún no han desarrollado una respuesta inmune, pudiendo progresar a una enfermedad tuberculoide o lepromatosa (7). Clínicamente se presenta con una o más lesiones tipo macula, hipopigmentadas, qué pueden sanar solas, indurarse o evolucionar a otras presentaciones de enfermedad indeterminada $(4,6)$. Incluso en países endémicos, los pacientes pueden pasar desapercibidos y ser diagnosticados como tiña versicolor, pitiriasis alba asociada a atopia, vitíligo, o hipopigmentación postinflamatoria asociada a eccema (4). Cuando la enfermedad evolucionada a etapas más avanzadas se caracteriza por la presencia de lesiones a nivel de SNP, como disminución de la sudoración y/o perdida de la termosencibilidad, con sensibilidad al dolor conservada (4). El diagnóstico de la lepra indeterminada es difícil (4) tanto clínica como molecularmente ya que su detección en medios biológico es extremadamente rara.

\section{Lepra tuberculoide / paucibacilar (TT)}

Respuesta inmunológica importante, mediada por células $T$, que determina un curso de la enfermedad menos severo (7) Lesiones paucibacilares de tipo macula, hipopigmentadas, con pérdida de la sensación térmica, táctil y al dolor, alargamiento nervioso que puede sanar solo, persistir o avanzar. En pieles claras las lesiones tienden a ser eritematosas y en pieles oscuras pueden apreciarse hiperpigmentadas. Secas y escamosas (por anhidrosis), lampiñas, hipo o anestésicas, con muy pocos bacilos. Algunas lesiones pueden tener bordes elevados con cicatrización central o diseminación periférica. Dolor y edema alrededor de la lesión que progresa con perdida sensorial y motora. El daño nervioso puede presentarse con áreas anestésicas o sin ninguna manifestación cutánea.

Figura 1. Imagen clínica de la lepra tuberculoide

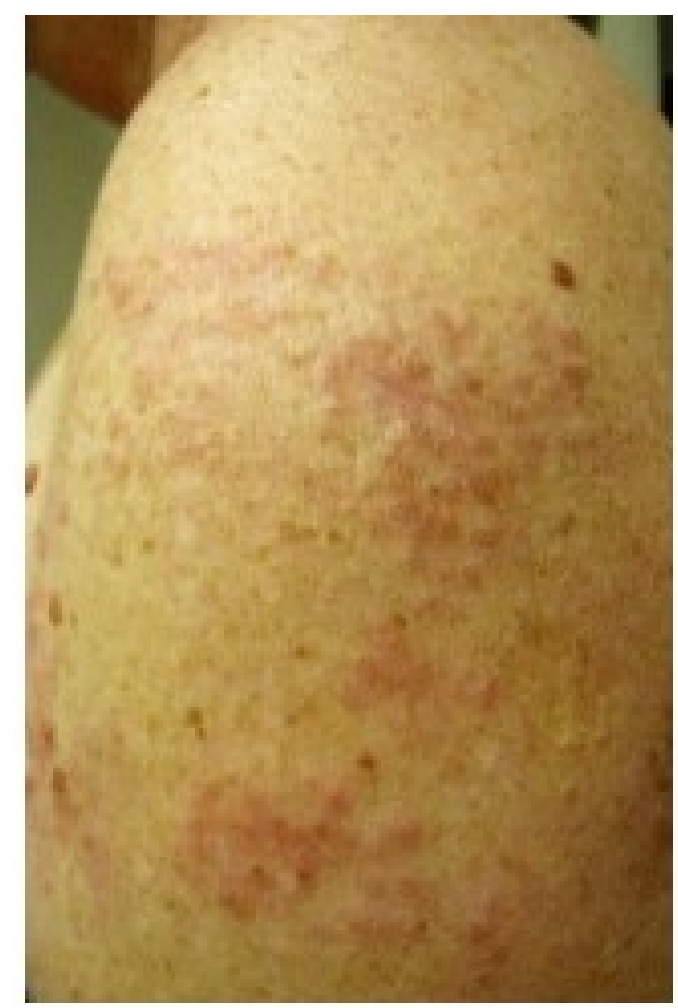

Fuente. NHDP Guide to the Management of Hansen's Disease.

\section{Lepra lepromatosa / multibacilar (LL)}

Se conoce históricamente como la lepra de Lucio en América Central y Sur, caracterizada por una infiltración difusa de la piel de la cara y las manos, con una apariencia suave y eritematosa, conociéndose también como "lepra bonita" (7).

De 20 a 200 lesiones diseminadas, simétricas, eritematosas a violáceas (7) tipo nódulos, infiltrados, múltiples de color rojocafé mejor llamados: lepromas (4).

Los sitios más comunes de aparición son cara y orejas, la distribución centro facial simétrica es conocida como facies leonina, 
asociada a la perdida de vello de las cejas y pestañas (madarosis). La afectación de la mucosa nasal puede llevar a la destrucción del tabique nasal y generar la presentación clínica de nariz en "silla de montar", llegando incluso a extender a nasofaringe, generando ulceras a nivel de paladar y laringe (4).

La afectación temprana de los nervios es asintomática a medida que avanza la enfermedad, los nervios dorsales periféricos se vuelven agrandando con anestesia posterior de las manos y los pies con patrón de "calcetín y guante" (7).

Una de las complicaciones más temidas, asociada a la forma multibacilar es la afectación ocular, que puede evolucionar a ceguera, se presenta en un $10 \%$ de los pacientes y fisiopatológicamente su causa es multifactorial (4).

La diseminación linfática o hematógena del M. leprae puede generar afectación de los riñones (glomerulonefritis con síndrome nefrótico subsecuente y amiloidosis), a nivel hepático puede generar hepatitis y fibrosis peri portal.

Los pacientes con LL, aparentemente carecen de una respuesta celular-inmune al complejo $M$. leprae, lo que lleva a una enfermedad generalizada y numerosas lesiones que pueden afectar a múltiples sistemas orgánicos, incluidos riñones y testículos (7).

Estado indeterminado (lepra borderline o lepra dismórfica):

La presentación clínica se basa en un estado indeterminado que puede variar entre lepromatoso y tuberculoide. La clasificación de Ridley - Jopling divide la lepra indeterminada en 3 subgrupos según el estado inmunológico del huésped (ver figura 2) (4).

\section{Lepra limítrofe tuberculoide (BT)}

En comparación con la lepra tuberculoide presenta un mayor número de lesiones y en proporción más extensa, afectación nerviosa motora y sensitiva, pero en menor proporción.

Se detectan pocas micobacterias. Lesiones eritematosas con bordes eritematosos bien delimitados, asimétricas, en ocasiones con presencia de lesiones satélite (ver figura 3) (4).

\section{Lepra limítrofe media (BB)}

Aumento del número de las lesiones, distribuidas simétricamente y pueden presentar características lepromatosas y tuberculoides. Se desarrolla afectación importante de folículos pilosos y glándulas sudoríparas. Dependiendo de la lesión, pocas y muchas bacterias pueden detectarse. Los nervios periféricos asocian déficits motores y sensitivos (4).

\section{Lepra limítrofe lepromatosa (BL)}

Lesiones tipo pápulas, placas, infiltradas, poco delimitadas, hipopigmentadas, distribuidas simétricamente.

El crecimiento de cabello y la sudoración se afectan importantemente.

Desarrollan extensa afectación del SNP y pueden encontrarse numerosas bacterias en los estudios (ver figura 4) (4).

\section{Reacciones leprosas}

Hasta un $30 \%$ de los pacientes con lepra desarrollan exacerbaciones conocidas como reacciones de lepra y son el significado de un equilibrio inmunológico alterado (4).

Las mismas pueden generarse de forma espontánea, durante el tratamiento e incluso posteriormente a un tratamiento exitoso (4).

\section{- Reacción tipo 1}

Pato fisiológicamente se describe como una rección de hipersensibilidad al $M$. leprae en la que se desarrolla un inicio abrupto de lesiones urticarias edematosas, sobre las lesiones leprosas de la piel.

Se asocia la presencia de abscesos a nivel de los nervios y puede presentarse asociado a fiebre alta. 
Ocurre incluso posterior a 12 meses del inicio del tratamiento (4).

\section{- Reacción tipo 2 (Eritema nodoso} leproso) (4).

Complicación inflamatoria grave de la lepra lepromatosa BL y LL, afecta a un $10 \%$ y $50 \%$ de las personas respectivamente, también se considera como factor de riesgo para su desarrollo, un índice bacteriano de 4+, ENL puede ocurrir antes, durante o después de la finalización exitosa de la terapia polifarmacológica.

Causa inflamación en muchos sistemas y se caracteriza por dolor intenso, sensibilidad cutánea (12) con la aparición de lesiones de tipo nódulos cutáneos-subcutáneos, eritemato violaceos, dolorosos. Que pueden encontrarse a lo largo de todo el cuerpo. Las lesiones en piel pueden ulcerarse y evolucionar a necrosis. Ocurren principalmente en las regiones extensoras de las extremidades, en la cara, el tronco (4).

- Reacción tipo 3 (fenómeno de Lucio) Caracterizada por parches violáceos e infiltrados bullosos. Ocurre en pacientes con enfermedad lepromatosa no tratada. Histopatológicamente vasculitis extensa con proliferación endotelial (4).

Figura 2. Imágenes clínicas de la lepra lepromatosa
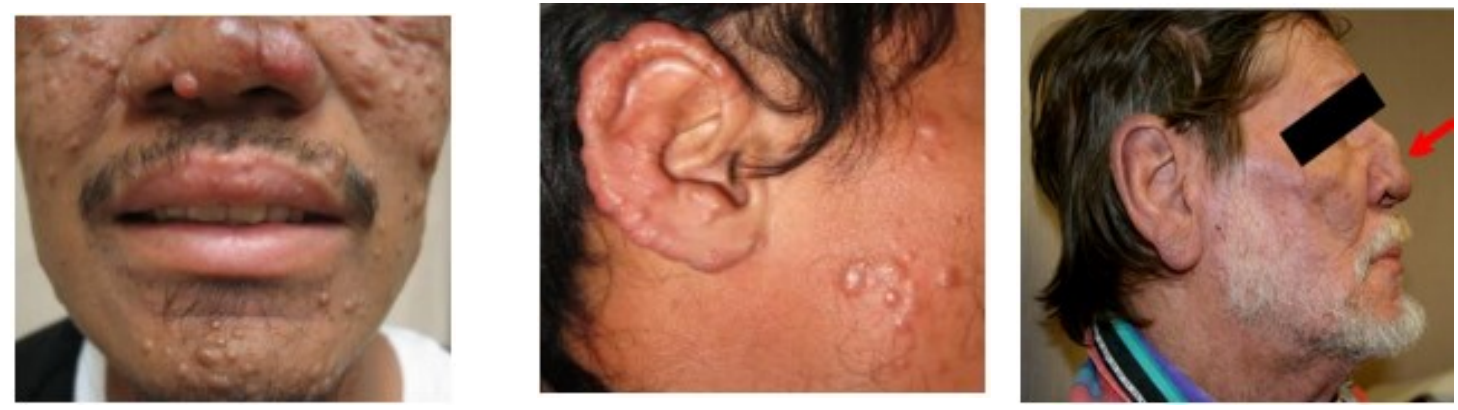

Fuente. NHDP Guide to the Management of Hansen's Disease

Figura 3. Imágenes clínicas de la lepra limítrofe tuberculoide
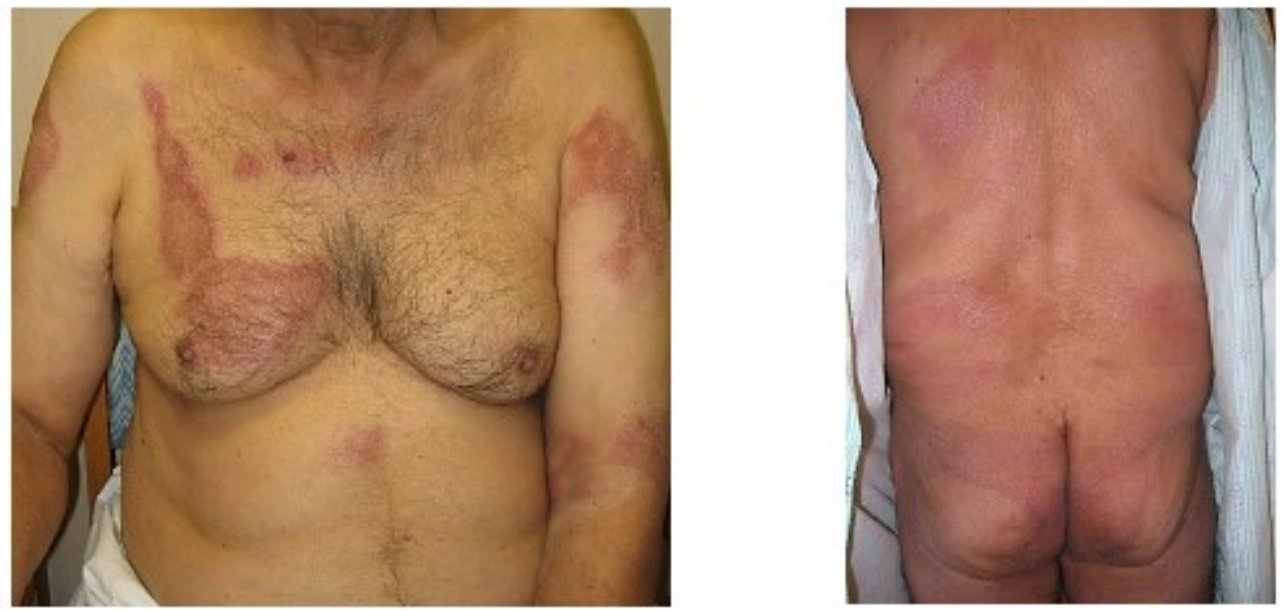

Fuente. NHDP Guide to the Management of Hansen's Disease 
Figura 4. Imágenes clínicas de la lepra limítrofe lepromatosa
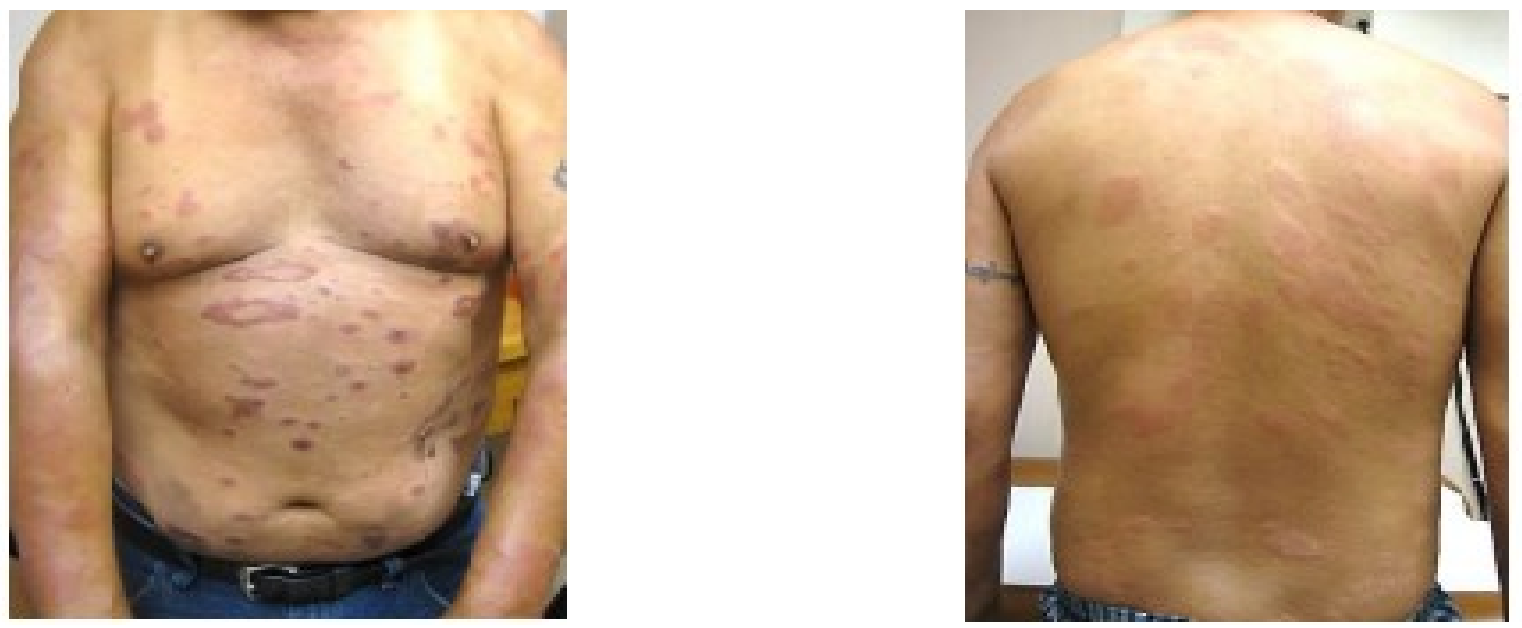

Fuente. NHDP Guide to the Management of Hansen's Disease

\begin{tabular}{|c|c|c|c|c|c|}
\hline \multirow{2}{*}{$\begin{array}{l}\text { Inmunidad } \\
\text { celular } \\
\text { Ridley- } \\
\text { Jopling } \\
\text { clasificación } \\
\end{array}$} & \multicolumn{5}{|c|}{ Rango desde alto (izquierda) a bajo (derecha) } \\
\hline & TT & BT & BB & $B L$ & LL \\
\hline $\begin{array}{l}\text { Descripción } \\
\text { de la lesión }\end{array}$ & $\begin{array}{l}\text { Maculas y } \\
\text { placas bien } \\
\text { definidas }\end{array}$ & $\begin{array}{l}\text { Maculas y } \\
\text { placas } \\
\text { infiltradas }\end{array}$ & $\begin{array}{l}\text { Lesiones } \\
\text { anulares con } \\
\text { bordes poco } \\
\text { definidos }\end{array}$ & $\begin{array}{l}\text { Lepromas y } \\
\text { lesiones } \\
\text { anulares }\end{array}$ & $\begin{array}{l}\text { Maculas, } \\
\text { Pápulas y } \\
\text { nodos } \\
\text { infiltrados }\end{array}$ \\
\hline $\begin{array}{l}\text { Núm. de } \\
\text { lesiones }\end{array}$ & Única & $\begin{array}{l}\text { Única o } \\
\text { varias }\end{array}$ & Varias & Numerosas & Innumerables \\
\hline Distribución & Localizado & Asimétrico & Asimétrico & Simétrico & Simétrico \\
\hline $\begin{array}{l}\text { Superficie de } \\
\text { la Lesión }\end{array}$ & $\begin{array}{l}\text { Seco y } \\
\text { escamoso }\end{array}$ & Seco & Algo brillante & Brillante & Brillante \\
\hline $\begin{array}{l}\text { Crecimiento } \\
\text { de Cabello } \\
\text { en la lesión }\end{array}$ & Ninguno & Disminuido & Algo & Levemente & Inafectado \\
\hline Sensibilidad & $\begin{array}{l}\text { Ausente } \\
\text { sobre las } \\
\text { placas }\end{array}$ & $\begin{array}{l}\text { Ausente } \\
\text { sobre las } \\
\text { placas }\end{array}$ & $\begin{array}{l}\text { Moderadamente } \\
\text { disminuida }\end{array}$ & $\begin{array}{l}\text { Levemente } \\
\text { disminuido }\end{array}$ & $\begin{array}{l}\text { No afectación } \\
\text { temprana }\end{array}$ \\
\hline $\begin{array}{l}\text { Índice } \\
\text { Bacilar }\end{array}$ & $\begin{array}{l}1+(0 \text { Bacilos } \\
\text { en } 100 \\
\text { campos })\end{array}$ & $\begin{array}{l}1-2+(1-10 \\
\text { Bacilos en } \\
100 \text { campos })\end{array}$ & $\begin{array}{l}2-3+(1-10 \text { bacilos } \\
\text { en } 10 \text { campos })\end{array}$ & $\begin{array}{l}3-4+(1-10 \\
\text { bacilos en } \\
\text { cada campo) }\end{array}$ & $\begin{array}{l}4-6+(100-1000 \\
\text { bacilos en } \\
\text { cada campo })\end{array}$ \\
\hline $\begin{array}{l}\text { OMS } \\
\text { clasificación }\end{array}$ & \multicolumn{2}{|c|}{ Paucibacilar } & & \multicolumn{2}{|c|}{ Multibacilar } \\
\hline \multicolumn{6}{|c|}{$\begin{array}{l}\text { TT: lepra tuberculoide, BT: tuberculoide limítrofe, BB: lepra limítrofe, BL: lepromatosa limítrofe, LL: lepra } \\
\text { lepromatosa }\end{array}$} \\
\hline \multicolumn{6}{|c|}{$\begin{array}{l}\text { Fuente. Traducido por Ana Victoria Vargas, tomado de: Lepra: aspectos clínicos y técnicas diagnósticas, Revista } \\
\text { de la Academia Estadounidense de Dermatología }\end{array}$} \\
\hline
\end{tabular}


Una de las principales limitantes en el diagnóstico de la enfermedad de Hansen es el simple hecho de no pensar en ella como posible diagnostico diferencial.

Es importante realizar una historia clínica detallada, historia de viajes recientes a zonas endémicas y factores involucrados que nos orientan a pensar en la enfermedad. La enfermedad de Hansen es un gran imitador y puede presentarse con una variedad de manifestaciones (11).

Podemos desarrollar una fuerte sospecha cuando la lesión presenta más de una de las siguientes características 0 signos cardinales $(7,3)$ :

1. Perdida de la sensibilidad en una lesión hipopigmentada o parche de piel rojiza.

2. Un nervio periférico engrosado o agrandado, con pérdida de la sensibilidad $y /$ o debilidad de los músculos inervados por ese nervio.

3. La presencia de bacterias acidorresistentes en un examen de SS.

La presencia de una de estas características nos orienta a pensar en el diagnóstico, y la presencia de las tres características tiene una especificidad diagnostica del 97\% (7).

La afectación nerviosa de la enfermedad se limita al SNP, principalmente a los nervios cubital y peroneo común y sin quedar limitados a estos, pueden verse afectados los nervios periféricos principales (7).

\section{Importancia del reconocimiento en la práctica clínica}

"I Wasted 3 Years, Thinking It's Not a Problem" (perdí 3 años, pensando que no es un problema) (13).

La enfermedad de Hansen es una de las discapacidades prevenibles más altas del mundo (13), su retraso en el diagnostico produce secuelas en aproximadamente 2 millones de personas en todo el mundo (10).
El retraso en el diagnóstico en conjunto con las discapacidades sigue siendo un importante desafío para la salud pública, cuanto mayor sea la demora, mayor es la probabilidad de riesgo de daño nervioso con discapacidad posterior. Debido a esto la familiarización con la enfermedad y la educación continua en su presentación clínica, permite la identificación temprana en aquellas personas con mayor riesgo de desarrollar discapacidades físicas sumado al empleo de campañas de educación destinadas a promover la consulta temprana y prevención de discapacidades físicas (10).

\section{CONCLUSIONES}

La enfermedad de Hansen es un desafío diagnostico para los médicos, debido a los múltiples subtipos clínicos de la enfermedad, pero aún más a su gravedad y a las posibles complicaciones desfigurantes (14), por lo que es de suma importancia el reconocimiento 0 sospecha de la enfermedad, permitiendo así un diagnóstico y tratamiento temprano de la misma, desafortunadamente esto sigue siendo un problema, un diagnóstico tardío o erróneo contribuye a la evolución de la enfermedad y al desarrollo de discapacidades irreversibles (15). La transmisión de persona a persona no es la única forma de adquirir la enfermedad, existen factores ambientales y animales que pueden desempeñar un papel en la persistencia de la misma, pero hace falta mucho por esclarecer y no es posible aun lograr una reducción o eliminación de la enfermedad desde el ámbito de la trasmisión humana (16) hasta no determinar las diferentes vías de transmisión y los mecanismos involucrados, de ahí la importancia del estudio de la enfermedad de Hansen y su reconocimiento temprano.

\section{Los autores declaran no tener conflicto de interés.}




\section{REFERENCIAS}

1. Ploemacher, T., Faber, WR, Menke, H., Rutten, V. y Pieters, T. (2020). Reservorios y vías de transmisión de la lepra; Una revisión sistemática. PLOS Enfermedades tropicales desatendidas, 14 (4), e0008276. https://doi.org/10.1371/journal.pntd.0008276

2. Rivera CA, Espinoza AA. Comportamiento epidemiológico de la lepra en Costa Rica, de 2012 al 2017 . Rev Clin Esc Med. 2018:8(6):28-36.

3. OMS, Directrices para Diagnóstico, tratamiento y prevención de la lepra, OPS. https://apps.who.int/iris/handle/10665/274127

4. Fischer, M. (2017). Lepra: descripción general de las características clínicas, el diagnóstico y el tratamiento. JDDG: Journal Der Deutschen Dermatologischen Gesellschaft, 15 (8), 801-827 https://doi.org/10.1111/ddg.13301

5. Sarode, G., Sarode, S., Anand, R., Patil, S., Jafer, M., Baeshen, H. y Awan, KH (2019). Aspectos epidemiológicos de la lepra. Enfermedad al mes, 100899.

https://doi.org/10.1016/i.disamonth.2019.100899

6. Mungroo, MR, Khan, NA y Siddiqui, R. (2020). Mycobacterium leprae: patogenia, diagnóstico y opciones de tratamiento. Patogenia microbiana, 104475.

https://doi.org/10.1016/j.micpath.2020.104475

7. Maymone, MBC, Laughter, M., Venkatesh, S., Dacso, MM, Rao, PN, Stryjewska, BM,... Dunnick, CA (2020). Lepra: aspectos clínicos y técnicas diagnósticas. Revista de la Academia Estadounidense de Dermatología. https://doi.org/10.1016/j.jaad.2019.12.080

8. Ministerio de salud de Costa Rica, Dia Mundial contra la Lepra: 26 de enero "Estigma y Derechos Humanos de las personas afectadas por la lepra" www.ministeriodesalud.go.cr

9. WHO, Estrategia mundial para la lepra 2016. 2020.Acelerar la acción hacia un mundo sin lepra. Disponible en: https://apps.who.int/iris/bitstream/handle/10665/2 08824/9789290225195-es.pdf

10. De Paula, HL, de Souza, CDF, Silva, SR, MartinsFilho, PRS, Barreto, JG, Gurgel, RQ,... Santos, VS (2019). Factores de riesgo de discapacidad física en pacientes con lepra https://doi.org/10.1001/jamadermatol.2019.1768

11. Martin, RD, Gomez, IF y Spies, LA (2017). Carga de la lepra. The Journal for Nurse Practitioners, 13 (8), 538-545. doi: 10.1016 / j.nurpra.2017.06.010 https://doi.org/10.1016/j.nurpra.2017.06.010

12. Walker, SL, Ventas, AM, Butlin, CR, Shah, M., Maghanoy, A.,... Lambert, SM (2017). Una escala de gravedad clínica de la lepra para el eritema nudoso leproso: un estudio de validación multicéntrico internacional de la Escala de gravedad ENLIST ENL. PLOS Enfermedades tropicales desatendidas, 11 (7), e0005716. https://doi.org/10.1371/journal.pntd.0005716

13. Muthuvel, T., Govindarajulu, S., Isaakidis, P., Shewade, HD, Rokade, V., Singh, R. y Kamble, S. (2017). "Perdí 3 años pensando que no era un problema": Retrasos del paciente y del sistema de salud en el diagnóstico de lepra en la India: un estudio de métodos mixtos. PLOS Enfermedades tropicales desatendidas, 11 (1), e0005192. https://doi.org/10.1371/journal.pntd.0005192

14. Souyoul, S., Saussy, K., Stryjewska, BM y Grieshaber, E. (2017). Lepra que imita el carcinoma de células basales en un paciente en tratamiento con fingolimod. Informes de casos de JAAD, $\quad 3 \quad$ (1), 58-60 https://doi.org/10.1016/j.jdcr.2016.11.005

15. Miyashiro, D., Cardona, C., Valente, NYS et al. Úlceras en pacientes con lepra, una manifestación clínica no reconocida: reporte de 8 casos. BMC Infect Dis 19, $1013 \quad$ (2019). https://doi.org/10.1186/s12879-019-4639-2

16. Ministerio de salud de Costa Rica, Dia Mundial contra la Lepra: 26 de enero "Estigma y Derechos Humanos de las personas afectadas por la lepra" www.ministeriodesalud.go.cr

17. NHDP Guide to Management por Hansen's Disease disponible en: https://www.hrsa.gov/sites/default/files/hrsa/hans ens-disease/pdfs/hd-guide-management.pdf 